\title{
Replacement of Foot-And-Mouth Disease Virus Cattle Tongue Titration by In Vitro Titration
}

\author{
Aldo Dekker 1, Froukje van Hemert-Kluitenberg ${ }^{1}$, Anna H. Oosterbaan 2, Kimberly Moonen 2 and Laure Mouton ${ }^{3}$ \\ ${ }^{1}$ Wageningen Bioveterinary Research, Lelystad, The Netherlands; ${ }^{2}$ Boehringer-Ingelheim, Lelystad, The Netherlands; ${ }^{3}$ Boehringer-Ingelheim, Lyon, \\ France
}

\begin{abstract}
Titration of foot-and-mouth disease cattle challenge virus in cattle tongue has been the standard for many years in many countries, although titration in animals has been replaced by in vitro methods for all other applications. The objective of the analysis was the replacement of in vivo titration of cattle challenge virus by in vitro titration. Using data from 32 in vivo titration experiments together with the in vitro titration results of the same samples obtained by plaque count on primary lamb or pig kidney cells, as well as data from the virus isolation control chart used in the laboratory, we show that the reproducibility of the in vitro titration is much higher than that of the in vivo titration. The titer on primary kidney cells was on average $1.4 \log _{10}$ higher than the titer determined by titration in cattle tongue $(\mathrm{PFU} / \mathrm{ml}$ compared to bovine $I_{50} / \mathrm{ml}$ ), but the difference varied among different strains. The study also shows that the probability of infection in cattle tongue is high even when a lower challenge dose is used, which makes the variability between strains less important. Based on these results, we propose to change the standard dose for cattle challenge from $10^{4}$ bovine $\mathrm{ID}_{50}$ to $10^{5.4} \mathrm{PFU}$, and to replace the in vivo cattle tongue titration method with the in vitro titration method.
\end{abstract}

\section{Introduction}

Foot-and-mouth disease (FMD) is a contagious disease of cloven hoofed animals, for which vaccination is the best control option in densely populated livestock areas (Backer et al., 2009, 2012a,b). The vaccines must be of good quality. For quality control of the finished vaccine, both the World Organisation for Animal Health (OIE) manual for terrestrial animals (OIE, 2017) as well as the European Pharmacopeia (EDQM, 2017a) prescribe challenge experiments in FMD-vaccinated cattle. The OIE prescribes that 10,000 bovine $\mathrm{ID}_{50}$ (median bovine infectious dose; $\mathrm{BID}_{50}$ ) is used for challenge; the European Pharmacopoeia does not specify the method of titration of the virulent bovine virus. To determine the $\mathrm{BID}_{50}$, the virus used for challenge is titrated in cattle.

Titration in cattle tongue was described in the early days of FMD virology (Henderson, 1949) when in vitro cell culture was not available. In the report of the 1972 OIE conference on standardization of potency tests, many authors considered 10,000 $\mathrm{BID}_{50}$ the standard challenge dose (Anon., 1972). However, it is not clear when and why $10,000 \mathrm{BID}_{50}$ became the standard, especially as at the same conference it was reported that the potency of vaccines was similar for different challenge doses $\left(10,10,000\right.$ or $\left.1,000,000 \mathrm{BID}_{50}\right)$ (Terré et al., 1972).
Although in vivo titration of FMD virus (FMDV) has been replaced by in vitro titration for most applications, several FMD laboratories still use in vivo titration for cattle-passaged viruses intended for challenge. Until recently that was also the case in Wageningen Bioveterinary Research (WBVR, formerly known as Central Veterinary Institute) in Lelystad, The Netherlands. A quantitative relation between in vitro virus dose and infection by aerosol challenge has been reported before (French et al., 2002), but very limited data are available comparing the relation between in vitro dose and response to needle infection into the dermis of the tongue.

From a methodological point of view, it is necessary to standardize the dose used for challenge. For a standardized dose it is essential that the method used to determine the dose is robust and delivers reproducible results. From an ethical point of view, we should try to replace, reduce and refine animal experiments (EU, 2010). If animal experiments are performed, they should be as robust and reproducible as possible (https://www.nc3rs. org.uk).

In the present study, we analyze the reproducibility of cattle tongue titration from data collected over 30 years of cattle tongue titrations in comparison to the results obtained with the same samples using in vitro titration. The objective of the 
analysis was to determine whether in vivo titration of cattle challenge virus could be replaced by in vitro titration.

\section{Animals, materials and methods}

\section{Animals}

Wageningen Bioveterinary Research is licensed to perform animal experiments. Permission from the animal ethical committee was obtained for all experiments prior to performing the experiment, according to the Wet op de dierproeven of 1977 and its amendments applicable at the times the respective experiments were performed (current license number AVD401002015265).

Conventionally reared, healthy cattle (common Dutch breeds, mainly Holstein Friesian, Friesian, MRY and Belgian blue or crossbreeds of those breeds, more than $90 \%$ female, $6-9$ months old) were used. The cattle were bought by an animal provider from a Dutch farm. The cattle were individually uniquely identified.

The cattle were housed together in a tie-stall with rubber matting as bedding and were fed pelleted grass supplemented with pelleted oat husks with ad-lib water present. In the more recent experiments the cattle were provided with salt licks to fulfil licking needs, and a small amount of hay. The experiments were performed by a trained veterinarian.

\section{Medium ingredients}

The current supplier of minimal essential medium (MEM) and antibiotics is Gibco (USA), and DMSO, amido-black and fetal bovine serum (FBS) are supplied by Sigma-Aldrich (USA), but in older experiments details on producers were not recorded.

\section{Viruses}

In vivo titration data were available for the 27 strains (the names of the strains are proprietary knowledge) within serotype A $(n=10), O(n=6), C(n=3)$, Asia1 $(n=3)$, and SAT2 $(n=5)$. For 3 strains a challenge virus batch was produced twice, once for the same producer because the titer of the first batch was too low, and twice for two different producers; thus, a total of 30 different batches were tested. Cattle challenge viruses were obtained by passage of the original FMDV isolate (without cell passage, or only 1 or 2 cell passages) in cattle tongue. On approximately 20 sites $0.1 \mathrm{ml}$ of the isolate, diluted between 10 and 1,000-fold in MEM supplemented with 2\% FBS and antibiotics, was injected into the upper epidermal/dermal layer of the tongue of a cow under anesthesia. The cow used for passage was euthanized by captive bolt and exsanguinated when a vesicle presented (mostly after 26-28 hours). The vesicular material was collected and a suspension was made in MEM supplemented with 2\% FBS and antibiotics using sterile sand and a pestle and mortar. The cattle challenge virus was stored at $-70^{\circ} \mathrm{C}$ until use.

\section{Cattle tongue titration}

Data were available from 32 experiments performed to determine the in vivo titer of the challenge virus at Wageningen Bioveteri- nary Research in Lelystad, The Netherlands, using 63 cattle (one titration was performed in only $1 \mathrm{cow}$ ) for the testing of 30 different batches of FMD cattle challenge virus belonging to $5 \mathrm{FMD}$ serotypes (A, O, C, Asia1, and SAT2).

For each standard cattle tongue titration, two cattle were randomly assigned to receive either the low virus doses or the high virus doses. Two cattle were chosen to allow for a sufficient range of dilutions to determine the titer. The experiment was not blinded because the observer was the same person who did the inoculation. Both cattle were anesthetized in the morning in the stable by intravenous injection of Xylazine $(0.2 \mathrm{mg} / \mathrm{kg} \mathrm{BW}$, currently Sedamun ${ }^{\circledR}$, Dechra, The Netherlands). In both cattle, the part of the tongue between the lingual fossa and the tip was marked into three equal sections by intradermal lingual injection of ink (East Indian ink, Talens, The Netherlands) using a $0.7 \times 32 \mathrm{~mm}$ hypodermic needle. In total, 4 dilutions of the challenge virus were tested (normally the dilutions $10^{-6}, 10^{-5}, 10^{-4}$, and $10^{-3}$ in MEM supplemented with $2 \%$ FBS and antibiotics were tested). The three highest dilutions were tested on the tongue of the first cow and the three lowest dilutions were tested on the tongue of the second cow. In each section of the tongue one dilution was tested by intradermal lingual injection into 7 spots of $0.1 \mathrm{ml}$ of test dilution using a $0.7 \times 32 \mathrm{~mm}$ hypodermic needle. In each cow the highest dilution was injected into the upper part of the tongue to avoid high concentrations of virus leaking out of the injection site onto the next injection site (see Fig. S1 ${ }^{1}$ ). In the last 8 experiments, both cattle were treated with non-steroidal anti-inflammatory drugs, initially with injectable drug (Ketofen 10\%, Boehringer Ingelheim, The Netherlands), but in the last experiment with Fynadyne transdermal ${ }^{\circledR}$ (MSD, The Netherlands) at the time of infection using the dose prescribed by the manufacturer.

After approximately 24 hours both cattle were anesthetized in the stable intravenously using $0.2 \mathrm{mg} / \mathrm{kg}$ bodyweight of Xylazine (currently Sedamun ${ }^{\circledR}$, Dechra, The Netherlands) combined with $2 \mathrm{mg} / \mathrm{kg}$ of Ketamine (10\%, Alfasan, The Netherlands). The tongues were inspected and the primary FMD lesions were noted. At the same time, it was evaluated whether the cattle fulfilled the humane endpoint for early euthanasia (unable to stand), which never occurred.

After approximately 48 hours, both cattle were euthanized using an overdose of pentobarbital (Euthasol 40\%, AST Farma, The Netherlands) and the tongues were inspected at post-mortem. The number of injection sites that produced a vesicle were counted for each dilution and the titer, bovine $\mathrm{ID}_{50}$, was calculated using the non-parametric Spearman method (Spearman, 1908). The virus dilutions used for titration in cattle tongue were frozen at $-70^{\circ} \mathrm{C}$ and later tested by plaque count on primary porcine or ovine kidney cells, see below (Dekker, 1998; Bouma et al., 2004). For each injection site dilution, the in vitro titer in that specific dilution was calculated.

In vitro titration

For in vitro titration, the plaque count was determined in primary kidney cells derived from young, healthy (3-6 weeks of age)

\footnotetext{
1 doi:10.14573/altex.1712222s
} 
lambs or pigs, specifically euthanized, using an overdose of pentobarbital (Euthasol 40\%, AST Farma, The Netherlands), for the culture of the cells. The kidneys were brought to the laboratory and cells were separated by chopping the cortex into small pieces and treating them with $0.06 \%$ trypsin. When individual cells were separated, the cells were washed and suspended in MEM with Hank's Salts with 5\% FBS and antibiotics. The cells were then cultured at $37^{\circ} \mathrm{C}$ in 24 closed culture flasks $\left(300 \mathrm{~cm}^{2}\right.$, Falcon, USA). After approximately 1 week the cells were collected and suspended in medium containing 20\% FBS and 10\% DMSO and subsequently frozen in liquid nitrogen and stored until use.

Primary kidney cells were thawed quickly and suspended in MEM containing 5\% FBS and antibiotics. After spinning down the cells, they were suspended in the same medium and seeded in 6-well collagen coated plates (Corning, USA); at least 240 plates can be cultured from 1 set of kidneys. Previous studies in our laboratory had shown that titers on lamb and pig kidney cells were very similar. In the plaque count, $200 \mu \mathrm{l}$ of the dilutions of the sample was incubated for 1 hour at $37^{\circ} \mathrm{C}$ in a humidified atmosphere, before $2.5 \mathrm{ml}$ of MEM containing $2 \% \mathrm{FBS}$ and $1 \%$ methylcellulose and antibiotics was added to each well. Monolayers were stained after 1 or 2 days (depending on the plaque formation observed microscopically) using amido-black $(0.1 \%$ amido-black in $1 \mathrm{M}$ acetic acid, $0.09 \mathrm{M}$ sodium acetate, $10 \%$ glycerol). In wells with 1 to 50 plaques, the plaques were counted and the number of plaque forming units in the original suspension was calculated. Information on variation in the plaque count was obtained from the samples included in titration studies, as well as from the control chart of the positive control $\left(\mathrm{C}_{1}\right.$ Detmold challenge virus) used in virus isolation between 2004 to $2017(\mathrm{n}=228)\left(\mathrm{Tab} . \mathrm{S} 1^{1}\right)$.

\section{Statistics}

A logistic regression model (EDQM, 2017b) using a binomial error distribution and a logit link function was used to determine the titer of the virus in each experiment $(n=32)$ and for each cow $(n=60,2$ cattle were excluded as they did not show any lesion, and 1 because lesions were seen on all injection sites). In the logistic regression model, the result variable was "lesions formation", and possible explanatory variables were the in vitro dose injected, the experiment and the animal. Normality of the data was assessed by evaluating the normal Q-Q plot. All statistical analyses were performed in $\mathrm{R}^{2}$

\section{Results}

All cattle were healthy at the start of the experiments. In the last 8 experiments the cattle were treated with NSAIDs at the time of virus application as a refinement. There were no adverse events and only the in vitro titer sometimes indicated that different dilutions would have been preferable. The $\log _{10}$ dilutions $-6,-5,-4$, and -3 were tested in most experiments, but in some experiments other dilutions were also included.

All observations are plotted together with the estimated dose response curves for each experiment in Figure 1. The logistic re-
Fig. 1: Observations on in vivo response in relation to dilutions after injection of $0.1 \mathrm{ml}$ into the tongue The lines represent the estimated logistic regression lines for each experiment. Individual observations are slightly displaced randomly on the vertical axis to avoid overlap.

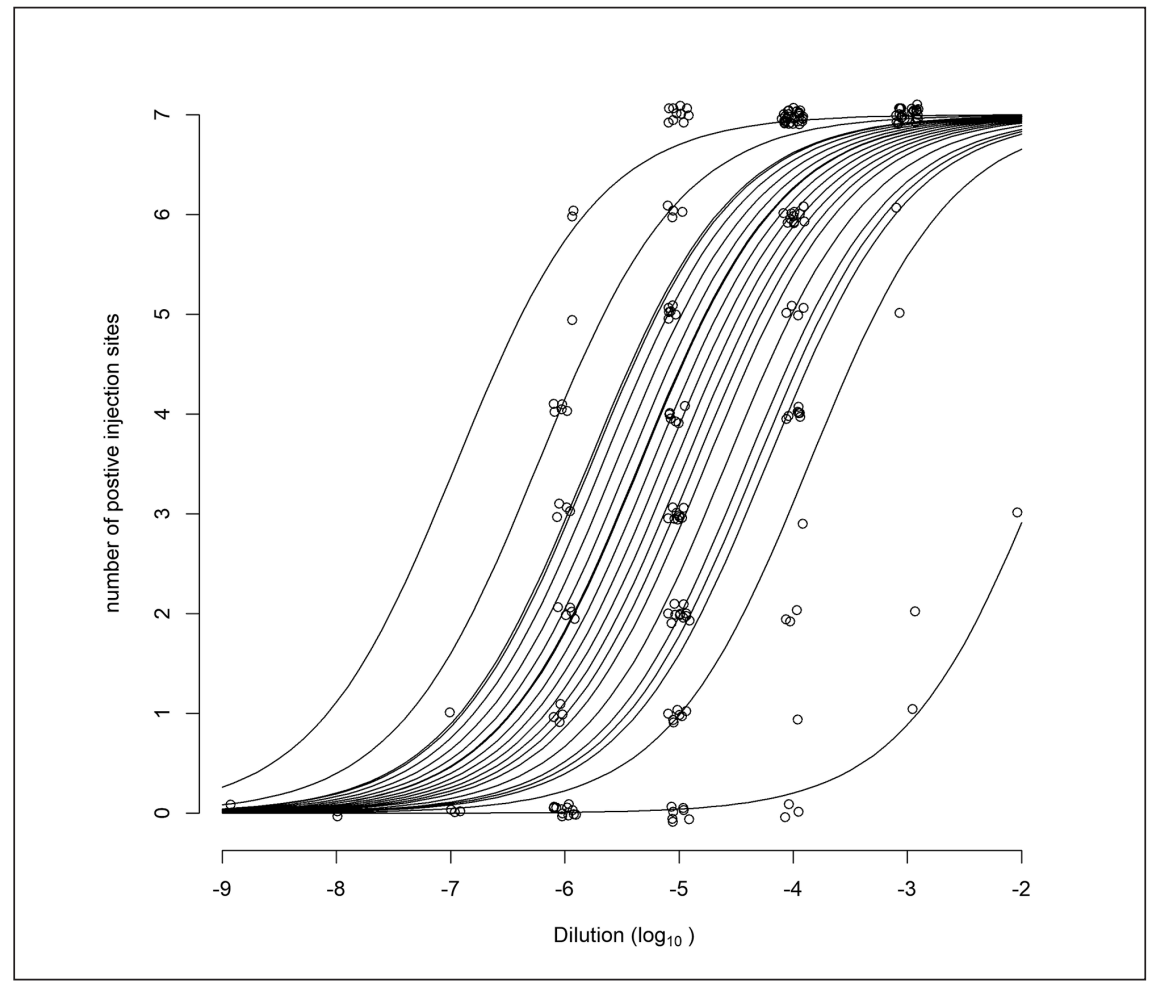

${ }^{2}$ R Core Team (2015). R: A language and environment for statistical computing. R Foundation for Statistical Computing, Vienna, Austria. http://www.R-project.org/ 


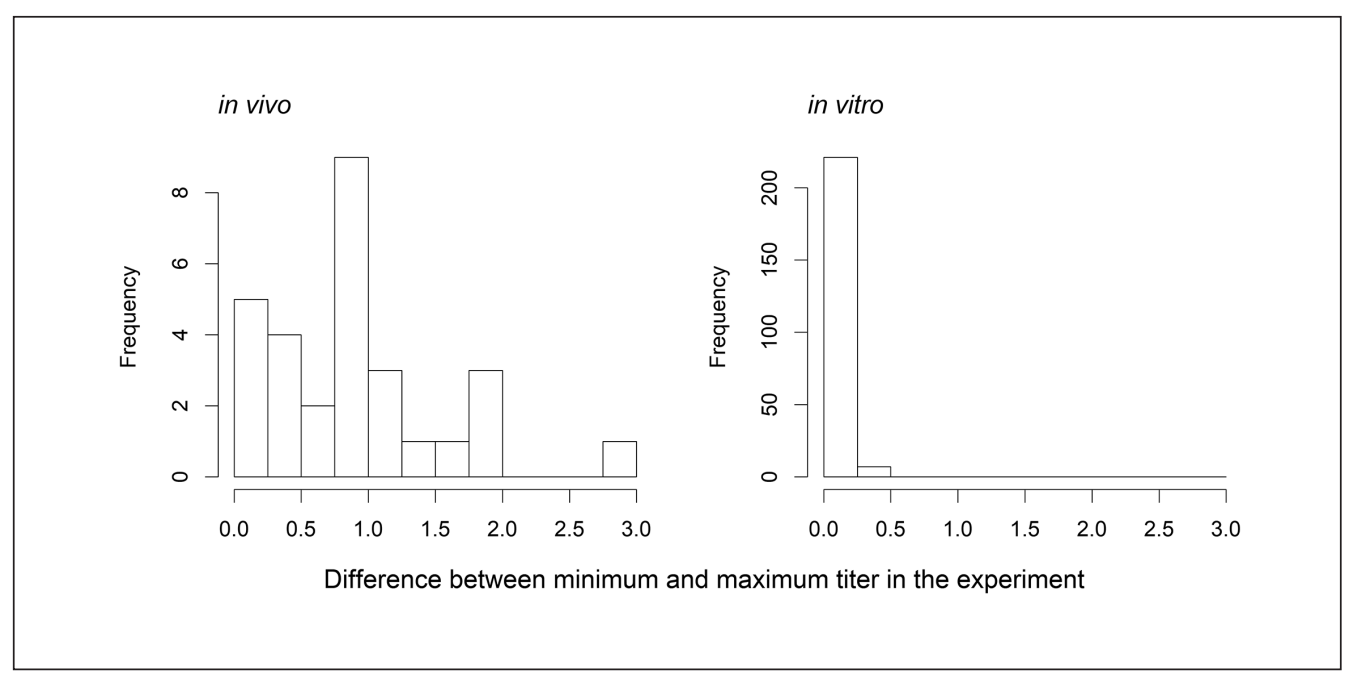

Fig. 2: Histograms of the difference between minimum and maximum titer observed in the same experiment, for both the in vivo titration and the results from duplicate control samples in in vitro titration

gression model with both in vitro dose and experiment as explanatory variables (the curves shown in Fig. 1) fitted significantly better than a model with only the in vitro dose as explanatory variable ( $\mathrm{p}<0.001$, likelihood ratio test). The logistic regression model with both in vitro dose and experiment was used to calculate the in vivo titer $\left(\mathrm{BID}_{50} / \mathrm{ml}\right)$ for each experiment. The logistic regression model with both in vitro dose and animal as explanatory variables fitted significantly better than a model with only the in vitro dose as explanatory variable $(\mathrm{p}<0.001$, likelihood ratio test). Therefore, the logistic regression model with both in vitro dose and animal was used to calculate the in vivo titer $\left(\mathrm{BID}_{50} / \mathrm{ml}\right)$ for each cow (cattle with lesions at all sites or no lesions at all were excluded). The AIC (Akaike information criterion) for the logistic regression model using in vitro dose and experiment was 668 , whereas the AIC for logistic regression model using in vitro dose and animal was 515 . The models using interaction were not further explored.

The between-experiment minimum, maximum, mean and standard deviation (SD) of the in vivo as well as in vitro titers were calculated (Tab. 1). The difference between in vivo and in vitro titration was on average $1.4 \log _{10}$, but the difference varied for different strains $\left(\mathrm{SD}=0.77 \log _{10}\right)$. The range in difference between in vivo and in vitro titration was 0.06-2.7. This shows that the analytical sensitivity of primary cells was always higher than the analytical sensitivity of a cattle tongue.

For analysis of the in vivo reproducibility (within test variation), the within-experiment differences of the 2 in vivo observations are shown in Figure 2. The within-experiment SD of the in vivo titration varied between 0 and 1.6, with a mean of 0.5 . Table 2 shows the results of the experiment (O Panasia 2 strain) where the largest

Tab.1: Summary statistics of titers found in various experiments and individual animals

\begin{tabular}{|l|l|l|l|l|}
\hline Titer & Minimum & Maximum & Mean & SD \\
\hline In vivo $\left(\log _{10} \mathrm{BID}_{50} / \mathrm{ml}\right)$ per experiment $(\mathrm{n}=32)$ & 2.8 & 8.0 & 6.0 & 0.9 \\
\hline In vivo $\left(\log _{10} \mathrm{BID} 50 / \mathrm{ml}\right)$ per animal $(\mathrm{n}=60)$ & 3.0 & 7.8 & 5.9 & 0.8 \\
\hline in vitro $\left(\log _{10} \mathrm{PFU} / \mathrm{ml}\right)$ & 5.2 & 8.5 & 7.4 & 0.8 \\
\hline
\end{tabular}

Tab. 2: Result of titration of FMD cattle challenge strain O Panasia 2

In this experiment the highest difference $\left(2.9 \log _{10}\right.$ PFU) between the $50 \%$ point in both cattle was observed.

\begin{tabular}{|l|l|l|l|}
\hline Dilution $\left(\log _{10}\right)$ & Titer injected $\left(\log _{10}\right.$ PFU per dose) & \multicolumn{2}{|l|}{ Positive sites (vesicular lesions) } \\
\cline { 3 - 4 } & & Cow 7372 & Cow 7373 \\
\hline-6 & 0.57 & NA & 2 \\
\hline-5 & 1.57 & 0 & 7 \\
\hline-4 & 2.57 & 2 & 7 \\
\hline-5 & 1.57 & 2 & NA \\
\hline
\end{tabular}


difference observed between 2 individual cattle was observed. For repeatability and reproducibility (between test variation) of the in vitro titration only very limited data were available for the challenge viruses; several were only titrated once. Therefore, the data of the control chart 2004-2017 was analyzed (Tab. S1 ${ }^{1}$ ). In total, 228 test data were available of duplicate titration of $\mathrm{C}_{1}$ Detmold challenge virus used as positive control in virus isolation in the laboratory. Figure 2 shows the observed differences between the 2 duplicates, the within test SD varied between 0 and $0.3 \log _{10}$ $\mathrm{PFU} / \mathrm{ml}$ with a mean of $0.1 \log _{10} \mathrm{PFU} / \mathrm{ml}$. Over a period of 13 years, the average titer of the positive control was $2.6 \log _{10} \mathrm{PFU} /$ $\mathrm{ml}$ with an SD of 0.3.

For 2 strains, $\mathrm{A}_{5}$ Westerwald and O/ITA/1993, the in vivo titration was repeated. For $\mathrm{A}_{5}$ Westerwald, the dilutions chosen were too high in the first experiment $\left(10^{-6}, 10^{-7}, 10^{-8}\right.$, and $\left.10^{-9}\right)$. In the repetition, dilutions $10^{-3}, 10^{-4}, 10^{-5}$, and $10^{-6}$ were tested. The estimated titers were $10^{6.3}$ and $10^{6.7} \mathrm{BID}_{50} / \mathrm{ml}$, respectively for the first and the second experiment. In the case of O/ITA/1993, the experiment was excluded from the reproduction analysis as the titration was repeated because a mistake was suspected in the first experiment.

Based on the model, the probability that one injection site becomes positive when injecting $5,000 \mathrm{BID}_{50}$ was $99.7 \%$. So, the probability that a cow develops a lesion on at least one site when injected with $10,000 \mathrm{BID}_{50}$ into two sites of the tongue (as recommended by the OIE manual), is $100 \%$. Using the logistic model with the in vitro titer and experiment as explanatory variables, the probability that an injection with $5.1 \log _{10}$ PFU results in a vesicular lesion is $97.7-100 \%$, so also $100 \%$ probability of developing at least one vesicular lesion when injected at two sites (which is a total of $5.4 \log _{10}$ PFU and based on the comparison of the mean in vivo titer and the mean in vitro titer similar to $10,000 \mathrm{BID}_{50}$ ). The use of 10,000 PFU (3.7 $\log _{10}$ per site) would result in a probability of infection of at least one of the 2 sites of $97-100 \%$.

\section{Discussion}

The objective of the analysis was the replacement of in vivo titration of cattle challenge virus by in vitro titration.

In the analysis of the in vivo titration data we used logistic regression as recommended by the European Pharmacopoeia (EDQM, 2017b). By combining results of all tests into one logistic regression model without interaction we assume a common slope for all experiments (Fig. 1). The model with both in vitro dose and experiment did fit better than the model with only in vitro dose. This shows that the relation between in vivo and in vitro virus titer is different for each strain. This confirms a previous finding that the sensitivity of in vivo and in vitro virus titration is different for different strains (House and House, 1989). Analysis of the SD of in vivo and in vitro titration (Tab. 1), which reflects mainly the difference in virus concentration in different samples, shows that the overall variation between both methods is similar.

The fact that the logistic regression model with both in vitro dose and animal has a lower AIC (value $=515$ ) than the logistic regression model with both in vitro dose and experiment (value $=668$ ) shows that the variation in response is better explained by differences in both strain and animal (which are not independent) than with difference in strain alone. Therefore, we also analyzed the in vivo between-animal variation and compared that with the variation found in in vitro titration. The maximum and mean of the within test SD is much larger in in vivo titration (Fig. 2). This shows that the in vitro titration has a higher reproducibility (within test variation). For the in vivo titration only one valid result of a repeated test was available, so it is not possible to draw any conclusions on reproducibility (between test variation) of the in vivo titration. For the in vitro titration it was easy to find data on reproducibility; an SD of $0.3 \log _{10}$ was found in the data from the virus isolation control chart. Based on these results, it can be concluded that the reproducibility (variation in results in the same test) of in vivo titration is lower than that of the in vitro titration.

Based on the low reproducibility of the in vivo titration as well as the methodological short-comings, in vitro titration of FMD cattle challenge virus is the preferred method. Although in vivo titration is probably not used a lot for other agents, it is advisable that a similar analysis is performed for these cases. It is likely that also in those instances an in vitro method would demonstrate a higher reproducibility.

The next question is the dose that should be used for a challenge test. Review of old literature did not reveal the reason for the choice of $10,000 \mathrm{BID}_{50}$; the analysis of the results, however, supports this choice as it shows that the probability that at least one vesicle is produced when injected at 2 sites is $100 \%$. So, the probability of control cattle developing FMD generalization is extremely high under the assumption that production of one vesicle is enough to produce generalization in control cattle. This assumption is probably largely true because the potency of 10 , 10,000 or $1,000,000 \mathrm{BID}_{50}$ has been shown to be very similar (Terré et al., 1972).

In our studies we observed a difference in mean titer between in vivo and in vitro values of $1.4 \log _{10}$. So, we propose that the best dose for infection would be $5.4 \log _{10}$ PFU $\left(5.1 \log _{10}\right.$ PFU per site), at which the overall probability of infection would also be $100 \%$ when injecting at 2 sites. Using a lower in vitro dose could lead to a slightly lower probability of infection of the control cattle, although still over $97 \%$ probability of infection of one of the 2 sites used for infection when using 10,000 PFU. Laboratories that cannot use primary cells should use a different dose as in some cells higher in vitro titers are seen than in primary cells, e.g., in LBFK- $\alpha$ V $\beta 6$ cells (LaRocco et al., 2013, 2015) or a lower in vitro titer than in primary kidney cells (House and House, 1989). Even though a high number of in vitro titrations can be performed with the kidneys of one animal, it would be better to use a continuous cell line for which the relation with the titer in primary cells is known.

The current study has a few limitations: There was only one valid repetition of the in vivo virus titration, which limits the analysis of in vivo reproducibility. Furthermore, the analysis of the titration in cattle tongue assumes that observations at different sites of the tongue are independent. However, as they are 
observed in the same animal, this assumption is not valid. It also assumes that all parts of the tongue are equally sensitive to FMD infection and lesions can be as easily formed at the back of the tongue as on the tip. This assumption is most likely also not valid. Dependence on the site of injection probably could explain the huge difference found between cattle. So, from a methodological point of view, in vivo titration is not a valid method, which supports the change to in vitro titration of challenge virus.

The analysis was performed to support changes to legislation and to replace titration in cattle tongue by in vitro titration. Based on the analysis, in vivo titration of FMD cattle challenge virus can be replaced by in vitro titration in primary kidney cells using a total of $5.4 \log _{10}$ PFU for intradermal injection into two sites of the tongue $(0.1 \mathrm{ml}$ per site).

\section{References}

Anon. (1972). XII Conférence de la commission de la Fièvre Aphteuse de OIE; normes de le contrôle d'efficacité des vaccins anti-aphteux. Bull Off Int Epizoot 77, 1131-1381.

Backer, J. A., Bergevoet, R. H. M., Hagenaars, T. J. et al. (2009). Vaccination Against Foot-And-Mouth Disease. Differentiating Strategies and their Epidemiological and Economic Consequences. Wageningen-UR. http://library.wur.nl/WebQuery/ wurpubs/fulltext/16086

Backer, J. A., Engel, B., Dekker, A. and van Roermund, H. J. (2012a). Vaccination against foot-and-mouth disease II: Regaining FMD-free status. Prev Vet Med 107, 41-50. doi:10.1016/j.prevetmed.2012.05.013

Backer, J. A., Hagenaars, T. J., Nodelijk, G. and van Roermund, H. J. (2012b). Vaccination against foot-and-mouth disease I: Epidemiological consequences. Prev Vet Med 107, 27-40. doi:10.1016/j.prevetmed.2012.05.012

Bouma, A., Dekker, A. and de Jong, M. C. M. (2004). No footand-mouth disease virus transmission between individually housed calves. Vet Microbiol 98, 29-36. doi:10.1016/j.vetmic. 2003.10.016

Dekker, A. (1998). Inactivation of foot-and-mouth disease virus by heat, formaldehyde, ethylene oxide and gamma radiation. Vet Rec 143, 168-169. doi:10.1136/vr.143.6.168

EDQM - European Directorate for the Quality of Medicines (2017a). Foot-and-mouth disease (ruminants) vaccine (inactivated) (Monograph 0063). European Pharmacopoeia 9.0.

EDQM (2017b). Statistical analysis of results of biological assays and tests (Chapter 5.3). European Pharmacopoeia 9.0.

EU (2010). Directive 2010/63/EU of the European parliament and of the council of 22 September 2010 on the protection of animals used for scientific purposes. Off J Eur Union L276, 33-79. http://data.europa.eu/eli/dir/2010/63/oj

French, N. P., Kelly, L., Jones, R. et al. (2002). Dose-response relationships for foot and mouth disease in cattle and sheep. Epidemiol Infect 128, 325-332. doi:10.1017/S0950268801006446

Henderson, W. M. (1949). The Quantitative Study Of FootAnd-Mouth Disease Virus. London: Her Majesty's Stationery Office.

House, C. and House, J. A. (1989). Evaluation of techniques to demonstrate foot-and-mouth disease virus in bovine tongue epithelium: Comparison of the sensitivity of cattle, mice, primary cell cultures, cryopreserved cell cultures and established cell lines. Vet Microbiol 20, 99-109. doi:10. 1016/0378-1135(89)90033-3

LaRocco, M., Krug, P. W., Kramer, E. et al. (2013). A continuous bovine kidney cell line constitutively expressing bovine $\alpha \mathrm{V} \beta 6$ integrin has increased susceptibility to foot-and-mouth disease virus. J Clin Microbiol 51, 1714-1720. doi:10.1128/ JCM.03370-12

LaRocco, M., Krug, P. W., Kramer, E. et al. (2015). Correction for LaRocco et al., A continuous bovine kidney cell line constitutively expressing bovine $\alpha v \beta 6$ integrin has increased susceptibility to foot-and-mouth disease virus. J Clin Microbiol 53, 755. doi:10.1128/JCM.03220-14

OIE (2017). Foot-and-mouth disease (infection with foot-andmouth disease virus). In OIE (eds.), Manual of diagnostic tests and vaccines for terrestrial animals. OIE, World Organisation for Animal Health. http://www.oie.int/fileadmin/Home/eng/ Health_standards/tahm/2.01.08_FMD.pdf

Spearman, C. (1908). The method of right and wrong cases (constant stimuli) without Gauss's formulae. British Journal of Psychology 2, 227-242. doi:10.1111/j.2044-8295.1908. tb00176.x

Terré, J., Chappuis, G., Perrenot, F. et al. (1972). Le contrôle d'activité sur bovins du vaccin anti-aphteux: Propositions pour des normes minimales. Bulletin-Office International des Epizooties 77, 1149-1174.

\section{Conflict of interest}

The authors declare the following competing financial interest(s): Laure Mouton, Anna H. Oosterbaan and Kimberly Moonen are employed by one of the vaccine producers that own the cattle challenge strains. The opinions expressed in this article are not informed by this affiliation. 